\title{
LÍMITES Y POTENCIALIDADES DE LA FORMACIÓN UNIVERSITARIA EN AMÉRICA LATINA PARA LA PRÁCTICA DE LA EXTENSIÓN RURAL*
}

\author{
FERNANDO LANDINI ${ }^{1}$
}

\begin{abstract}
RESUMEN: Los estudios sobre formación de extensionistas suelen focalizar en la implementación de capacitaciones, quedando en segundo plano la educación universitaria. Se analizaron los límites y potencialidades de la universidad para formar extensionistas a partir de entrevistas a 133 técnicos de diferentes países latinoamericanos. Los resultados muestran que la formación de grado en ciencias agropecuarias incluye contenidos insuficientes sobre extensión, la educación suele ser excesivamente teórica, y predomina un enfoque que simplifica en exceso la complejidad de los agroecosistemas. A la vez, se observa que los posgrados y diplomados en extensión y desarrollo rural poseen gran potencialidad. El artículo finaliza presentando diferentes propuestas para enfrentar las limitaciones identificadas.

Palabras-clave: Educación universitaria. Extensión rural. Posgrados. Agricultura.

\section{LIMITS AND POTENTIALITIES OF UNIVERSITY EDUCATION IN LATIN AMERICA FOR RURAL EXTENSION PRACTICE}

\begin{abstract}
Research on the education of extension workers tends to focus on the implementation of training activities, while studying the role of university education is infrequent. This research analyzes the limits and potentialities of university for educating extension workers based on 133 interviewees to practitioners from different Latin American countries. Results show that undergraduate university education in agricultural sciences includes insufficient knowledge on rural extension, tends to be excessively theoretical, and has an approach that oversimplifies the complexity of agroecosystems. In contrast, graduation studies and diplomas on extension and rural development have great potential. The article finishes presenting different proposals to overcome the identified limitations.
\end{abstract}

Keywords: University education. Rural extension. Graduation studies. Agriculture.

\footnotetext{
El presente estudio fue financiado por: 1) la Agencia Nacional de Promoción Científica de Ministerio de Ciencia, Tecnología e Innovación Productiva y la Universidad de la Cuenca del Plata en el marco del proyecto "Aprendizajes Colectivos y Reflexión sobre la Práctica en el Diálogo entre Extensionistas Rurales. Un Estudio de Caso Múltiple en los Países del Cono Sur” (PICT 2015-0692); y 2) el Consejo Nacional de Investigaciones Científicas y Técnicas en el marco del proyecto "Aprendizajes Colectivos y Reflexión sobre la Práctica en el Diálogo entre Extensionistas Rurales. Un estudio de Caso Múltiple Realizado en Argentina, Colombia, Cuba, Ecuador, Guatemala y Perú" (PIP 112-201501-00108-CO).

1.Consejo Nacional de Investigaciones Científicas y Técnicas - Universidad de la Cuenca del Plata - Posadas, Argentina. E-mail: landini_fer@hotmail.com
} 


\title{
LIMITES E POTENCIALIDADES DA FORMAÇÃO UNIVERSITÁRIA NA AMÉRICA LATINA PARA A PRÁTICA DA EXTENSÃO RURAL
}

\begin{abstract}
RESUMO: Os estudos sobre educação de extensionistas rurais normalmente focam na implementação de capacitações, deixando em segundo plano a educação universitária. Analisaram-se os limites e as potencialidades da universidade para formar extensionistas a partir de entrevistas realizadas com 133 técnicos de diferentes países latino-americanos. Os resultados mostram que a graduação em ciências agropecuárias inclui conteúdos insuficientes sobre extensão e costuma ser excessivamente teórica, além de predominar um enfoque que simplifica excessivamente a complexidade dos agroecossistemas. Por outro lado, observa-se que as pós-graduações em extensão e desenvolvimento rural têm potencial grande. $\mathrm{O}$ artigo finaliza apresentando diferentes propostas para enfrentar as limitações identificadas.
\end{abstract}

Palavras-chave: Educação universitária. Extensão rural. Pós-graduações. Agricultura.

\section{Introducción}

$\mathrm{L}$

a extensión rural (ER) constituye una práctica altamente compleja y diversa (SULAIMAN; DAVIS, 2012), que involucra desde apoyo productivo, organizacional y comercial a productores agropecuarios (BIANQUI et al., 2015) hasta la facilitación de procesos de articulación interinstitucional para el desarrollo rural desde una perspectiva territorial y sistémica (MOSCHITZ et al., 2015; TORRADO PORTO; CATULLO, 2017). Así, se delinea un escenario complejo en términos formativos, en tanto la ER excede los conocimientos y capacidades que ofrecen las distintas disciplinas y trayectos formativos de nivel terciario y universitario existentes en la actualidad.

En este marco, diferentes autores han destacado que la ER es por naturaleza interdisciplinaria (MULDER, 2012), por lo que requiere equipos conformados por profesionales de diferentes disciplinas (SULAIMAN; DAVIS, 2012; LANDINI, 2015). En paralelo, también se ha señalado la necesidad de fortalecer los conocimientos y capacidades de los extensionistas rurales, a fin de que estos puedan cumplir adecuadamente con su función (KHALIL et al., 2009; CHRISTOPLOS et al., 2012; CUEVAS REYES et al., 2014).

Ante esta situación, si bien existen autores que han subrayado el importante rol que juega la formación universitaria de los profesionales que luego se desempeñarán como extensionistas (MOVAHEDI; NAGEL, 2012; RAGASA et al., 2016), resulta claro que la discusión ha evolucionado centrándose progresivamente en la búsqueda de alternativas y opciones para fortalecer la formación en servicio de los extensionistas. En este sentido, existen numerosos trabajos en la literatura académica orientados a la identificación de necesidades de capacitación de los extensionistas (LANDINI, 2013; GOMBE et al., 2015) y a la evaluación de resultados o impactos de propuestas formativas (SINGH et al., 2010; SHARMA et al., 2016). Incluso, la literatura académica cuenta con diferentes artículos que presentan iniciativas de formación alternativa, caracterizadas en general por su énfasis reflexivo o por su profunda articulación con contextos reales de práctica (GBOKU; MODISE, 2008; LEFORE, 2015; LANDINI; BRITES, 2018).

En contraste, el foco puesto en la formación en servicio de los extensionistas parece haber dejado en un segundo plano la investigación relacionada con la formación universitaria (de grado y posgrado) de quienes llevan adelante tareas de extensión. Claro está, esto no significa que el tema no haya sido abordado o discutido, 
sino que usualmente se lo ha hecho, pero desde una perspectiva reflexiva o como tema secundario en el marco de investigaciones orientadas a identificar necesidades formativas para el diseño de capacitaciones en servicio. Así, por ejemplo, se ha señalado que la formación en ciencias agropecuarias a nivel universitario tiende a ser excesivamente teórica (MOVAHEDI; NAGEL, 2012; MOJARRADI; KARAMIDEHKORDI, 2016), a favorecer esquemas de pensamiento lineales que dificultan la comprensión de procesos sistémicos (SALOMONSSON et al., 2009; SARANDÓN; FLORES 2014), y a carecer de contenidos orientados al desarrollo de capacidades para interactuar con las personas (GBOKU; MODISE, 2008; MOVAHEDI; NAGEL, 2012).

En este contexto, el presente artículo se propone comprender la percepción de extensionistas rurales de diferentes países latinoamericanos sobre la relevancia y las limitaciones de la formación universitaria de grado y posgrado para el trabajo de ER a partir de sus trayectorias personales.

\section{Aportes Conceptuales para Pensar la Relación entre Teoría y Práctica}

A fin de reflexionar conceptualmente sobre los resultados de esta investigación se retoman dos líneas conceptuales que piensan el vínculo entre teoría y práctica en el marco de procesos educativos y de aprendizaje. La primera de ellas refiere a la teoría del aprendizaje experiencial. El aprendizaje experiencial pone el foco en los procesos de aprendizaje que dan en el marco de la participación en las actividades propias de la vida real (YARDLEY et al., 2012). En este marco, diferentes autores señalan el rol clave que juegan situaciones en las cuales se deben resolver conflictos o divergencias entre la teoría y la experiencia concreta (ORD, 2012), por ejemplo, cuando no se cuenta con conocimientos teóricos específicos para tomar una decisión o cuando se observan divergencias entre lo que la teoría indica y lo que la práctica muestra.

En el marco de la teoría del aprendizaje experiencial se reconoce que la reflexión sobre la experiencia (en un proceso de retroalimentación entre acción y reflexión) y ıa experimentación activa, ocupan un lugar central en los procesos de aprendizaje (KRAKAUER et al., 2017). En esta línea, siguiendo a diferentes autores, Ord (2012) describe cuatro momentos del aprendizaje experiencial, que construyen un ciclo que articula teoría y práctica: experiencia concreta, observación reflexiva, conceptualización abstracta y experimentación activa.

El enfoque de la Teoría de la Acción también contribuye a la comprensión de la articulación entre teoría y práctica. No obstante, a diferencia de la teoría del aprendizaje experiencial, ésta pone el foco en los procesos de aprendizaje que permiten desarrollar prácticas efectivas. La Teoría de la Acción sostiene que las personas actúan a partir de las teorías que tienen sobre cómo funciona la realidad, con el fin de alcanzar objetivos o metas que se proponen (ARGYRIS, 1991; PEIXOTO; PEREIRA, 2013). En términos prácticos, las teorías de la acción pueden ser entendidas como conjuntos articulados de conocimientos relativos a un área específica de la realidad.

En general, la Teoría de la Acción señala que las personas replantean sus estrategias de acción, y más aún, sus propias teorías, cuando aquellas que utilizan no les permiten alcanzar los resultados que se proponen (TAGG, 2010). De esta forma, se reconoce el potencial de la acción y de la reflexión sobre sus resultados prácticos como forma de ampliar, complejizar y perfeccionar los marcos teóricos que sustentan la práctica, en línea con las referencias presentadas relativas a la teoría del aprendizaje experiencial.

\section{Metodología}

Se llevó adelante una investigación cualitativa de carácter exploratorio descriptivo. Se realizaron 86 entrevistas (68 individuales y 18 grupales) a un total de 133 extensionistas que trabajan en seis diferentes países: 
Argentina, Chile, Cuba, Ecuador, Guatemala y Uruguay. La finalidad de las entrevistas fue comprender cómo los entrevistados aprendieron a ser extensionistas rurales, y cómo continúan aprendiendo a ser mejores en su labor. En este marco, ocupó un lugar central la identificación y caracterización de las principales fuentes de conocimiento, dentro de las cuales la educación formal juega un rol determinante. Las entrevistas fueron grabadas. Para asegurar que los tópicos centrales fueran abordados por igual en todas ellas, se diseñó un protocolo con preguntas y ejes temáticos.

Los países participantes fueron seleccionados por su accesibilidad, en particular por el vínculo del autor con instituciones de ER. En países con sistemas públicos de extensión de alcance nacional (Argentina, Chile, Ecuador y Guatemala), se optó por entrevistar a extensionistas que trabajaban en el marco de los Ministerios de Agricultura o su equivalente. En Argentina, las instituciones seleccionadas fueron el Instituto Nacional de Tecnología Agropecuaria (INTA) y la Subsecretaría de Agricultura Familiar (SsAF), en Chile el Programa de Desarrollo Local (Prodesal) del Instituto de Desarrollo Agropecuario (Indap), en Ecuador el Ministerio de Agricultura y Ganadería (MAGA), y en Guatemala el Ministerio de Agricultura, Ganadería y Alimentación (también MAGA). En Cuba se entrevistó a profesionales y técnicos de nivel municipal del Ministerio de Agricultura que trabajan en diferentes áreas y reparticiones, a extensionistas de institutos de investigación y a personal técnico de unidades productivas de carácter estatal o cooperativo. Por último, en el caso de Uruguay se entrevistó a asesores independientes, a técnicos de la Cooperativa Agraria Nacional y a extensionistas y personal técnico de diferentes instituciones públicas vinculadas con la producción agropecuaria. En la Tabla 1 se presenta información detallada de las diferentes muestras.

Tabla 1. Descripción de las diferentes muestra.

\begin{tabular}{|c|c|c|c|c|c|c|c|}
\hline & Argentina & Chile & Cuba & Ecuador & Guatemala & Uruguay & Total \\
\hline $\begin{array}{c}\text { Entrevistados } \\
\text { (\% mujeres })\end{array}$ & $25(32 \%)$ & $20(30 \%)$ & $21(33 \%)$ & $24(50 \%)$ & $22(32 \%)$ & $21(33 \%)$ & $133(35 \%)$ \\
\hline $\begin{array}{c}\text { Entrevistas } \\
\text { individuales/ } \\
\text { grupales }\end{array}$ & $11 / 4$ & $13 / 2$ & $8 / 3$ & $12 / 3$ & $12 / 3$ & $12 / 3$ & $68 / 18$ \\
\hline $\begin{array}{c}\text { Provincia o } \\
\text { departamento }\end{array}$ & Corrientes & $\begin{array}{c}\text { Región } \\
\text { Metropolitana }\end{array}$ & Granma & Cotopaxi & $\begin{array}{c}\text { Chiquimula } \\
\text { y Zacapa }\end{array}$ & $\begin{array}{c}\text { Paysandú } \\
\text { y Salto }\end{array}$ & - \\
\hline Institución & INTA y SsAF & PRODESAL & Varios & MAGA & MAGA & Varios & - \\
\hline Edad media & 45 años & 39 años & 51 años & 33 años & 36 años & 48 años & 42 años \\
\hline $\begin{array}{l}\text { Experiencia } \\
\text { media (años) }\end{array}$ & 12,2 años & 11,4 años & 17,9 años & 6,1 años & 8 años & 17,2 años & 12,5 años \\
\hline $\begin{array}{l}\text { \% de titulados } \\
\text { universitarios }\end{array}$ & $88 \%$ & $53 \%$ & $86 \%$ & $100 \%$ & $43 \%$ & $100 \%$ & $80 \%$ \\
\hline $\begin{array}{c}\% \text { egresados } \\
\text { agropecuarios }\end{array}$ & $60 \%$ & $53 \%$ & $71 \%$ & $96 \%$ & $35 \%$ & $85 \%$ & $67 \%$ \\
\hline $\begin{array}{l}\% \text { egresados cs. } \\
\text { sociales }\end{array}$ & $8 \%$ & $0 \%$ & $0 \%$ & $0 \%$ & $5 \%$ & $15 \%$ & $5 \%$ \\
\hline
\end{tabular}

Fuente: Elaboración propia. "Agropecuarios" incluye a universitarios con título de ingeniero agrónomo, veterinario, zootecnista o equivalente. "Cs. Sociales" incluye a universitarios con título de trabajador social y psicólogo. 
Más allá de la información presentada en la Tabla 1, debe reconocerse que los territorios en los que se realizó el estudio tienen diferentes características productivas, y que los extensionistas entrevistados en cada uno de ellos poseen diferente perfil. La provincia de Corrientes (Argentina) se caracteriza por la ganadería extensiva bovina, por el cultivo de cítricos, tabaco, arroz, yerba mate, té y algodón, y por la actividad forestal. La muestra incluye 13 ingenieros agrónomos, 5 licenciados en economía agropecuaria, 2 trabajadores sociales, 2 veterinarios y 3 no universitarios. La Región Metropolitana de Chile se encuentra marcada por la presencia de la ciudad de Santiago, capital y mayor ciudad del país, aunque las entrevistas fueron realizadas en municipios alejados con características rurales. A nivel agrícola se destaca la producción de hortalizas y frutales, favorecidas por la disponibilidad de riego. La muestra de extensionistas incluye 9 técnicos agrícolas, 6 ingenieros agrónomos, 3 veterinarios y 2 de otras profesiones.

La provincia de Granma (Cuba) se caracteriza por el cultivo de arroz y hortalizas, y por la producción de ganado vacuno (para leche), porcino y aviar. La muestra se compone de 10 ingenieros agrónomos, 4 veterinarios, 2 ingenieros agroindustriales y el resto profesionales y técnicos de otras disciplinas. En Ecuador el estudio fue realizado en la provincia de Cotopaxi, caracterizada por una gran variabilidad en altitud, que va desde los 250 hasta los 5.897 m.s.n.m, lo que diversifica las alternativas productivas según los pisos térmicos. Dentro de las actividades agropecuarias se destaca el cultivo de cebada, trigo, maíz, legumbres, hortalizas, banano, frutales y caña de azúcar, y la cría de ganado vacuno. Los entrevistados fueron 15 ingenieros agrónomos, 8 veterinarios y zootecnistas y 1 ingeniero agroindustrial.

Los departamentos de Chiquimula y Zacapa (Guatemala) se ubican en el corredor seco centroamericano y se caracterizan por niveles muy altos de pobreza y desnutrición crónica. A nivel agrícola se observa producción de maíz, poroto, mandioca, banano y tabaco, junto con grandes fincas de ganado vacuno. El perfil formativo de la muestra es altamente diverso, incluyendo 7 peritos o técnicos agrónomos, 6 ingenieros agrónomos y profesionales y técnicos de muy diferentes áreas. Por último, en Uruguay el estudio se llevó a cabo en los departamentos de Paysandú y Salto (región litoral norte). A nivel productivo la zona se caracteriza por la ganadería vacuna y ovina, y por el cultivo de frutales y hortalizas. De los entrevistados 13 son ingenieros agrónomos, 4 veterinarios, 2 psicólogos y el resto de otras profesiones.

Las entrevistas fueron analizadas de manera flexible a partir de los principios de la Teoría Fundamentada, siguiendo las recomendaciones de Taylor y Bodgan (1990). En concreto, se procedió a una primera lectura de las entrevistas para identificar todos los fragmentos que abordaran el rol de la educación universitaria (de grado y postgrado) en la práctica de extensión, o que pudieran ser utilizados para reflexionar sobre ella. En este proceso se identificaron los siguientes ejes temáticos, los cuales fueron utilizados para categorizar de manera exhaustiva todas las entrevistas con el apoyo del software para análisis cualitativo Atlas.Ti. Se indica entre paréntesis la cantidad de fragmentos categorizados dentro de cada eje temático:

- Experiencia rural previa o como productor (55);

- Aprendizaje en escuelas agrotécnicas (de nivel medio o terciario) (33);

- Aprendizaje en la universidad (225);

- Postgrados (43).

Luego se procedió a una lectura sistemática de los fragmentos correspondientes a cada uno de los cuatro ejes temáticos (utilizando la opción "familias" provista por el software), lo que permitió identificar y subcategorizar diferentes ideas o afirmaciones frecuentes dentro de cada uno de ellos. A continuación, se presentan de manera integrada las afirmaciones más frecuentes de los entrevistados, diferenciando entre países cuando se observan diferencias entre ellos. 


\section{Resultados}

Los resultados del estudio se desarrollan divididos en subtítulos. A fin de presentar evidencias de los resultados, se citan frases textuales de las entrevistas. Para una mejor identificación, luego de cada cita se indica un código compuesto por un número inicial, referido el número de entrevista dentro del país respectivo, las dos letras iniciales del país (“Ar|”, “Ch”, etc.), el carácter de la entrevista (“Gr” para grupal e "In" para individual), y las letras " $\mathrm{M}$ " o " $\mathrm{H}$ " para indicar si la persona es mujer u hombre. Así, el código “4.Ar-In-H” significará "Entrevista 4 de Argentina, de tipo Individual, Hombre”.

\section{Experiencias Familiares y Formación Técnica de Nivel Medio y Terciario}

Para comprender con mayor profundidad los límites y potencialidades de la formación universitaria para el trabajo de ER, resulta conveniente analizarla en contraste con otras fuentes de conocimiento previas (incluso alternativas), como son las experiencias personales infantiles y adolescentes relacionadas con la vida rural y la realización de estudios agropecuarios, de nivel medio o terciario.

Tanto en Argentina como en Chile, algo más de un tercio de los entrevistados hicieron referencia al valor de provenir de familia de agricultores familiares o de haber crecido en ambiente rural como base para su trabajo actual como extensionistas. En contraste, en países como Cuba, Ecuador y Uruguay, esto casi no fue mencionado, ubicándose Guatemala en una posición intermedia. Un entrevistado explica el valor para su trabajo de ser de familia de agricultores: "yo viví en predio durante mi niñez, [...] yo tengo manejo en animales, [...] en hortaliza, en frutales [...] sé injertar, he hecho labores de campo [...] No sólo lo que me enseñaron cuando estudié" (15.Ch-Gr-H).

Esta "ventaja formativa" de ser de familia de agricultores o de provenir de zonas rurales hace referencia a dos cuestiones centrales. La primera, apunta a contar con "conocimiento empírico [productivo] por ser pequeño productor" (13.Ar-Gr-H), mientras que la segunda corresponde a un conocimiento de la dimensión cultural de la gente de campo en general, como base para construir una buena relación con los agricultores: "[yo] conozco la idiosincrasia del lugar. Es importante conocer la idiosincrasia del lugar para llegar y entrar bien a la familia" (2.Ar-I-H).

Por otra parte, poco más de un tercio de los entrevistados provenientes de Chile y Guatemala hicieron referencia a la importancia de su formación técnica agropecuaria (sea de nivel medio o terciario) para su trabajo como extensionistas. Cabe destacar que en el resto de los países el tema no fue mencionado, lo que no resulta extraño si se tiene en cuenta que las muestras de ambos países tienen los menores porcentajes de extensionistas con título universitario. En este marco, conveniente diferenciar entre la formación técnicoproductiva de nivel medio o terciario como alternativa o como paso previo a la formación universitaria, ya que particularmente en Guatemala es frecuente que los Peritos Agrónomos de nivel medio estudien como ingenieros agrónomos mientras trabajan como extensionistas.

Quienes recibieron formación técnico-productiva (media o terciaria) antes de trabajar como extensionistas, suelen señalar que esto les permitió adquirir conocimientos productivos fundamentales para su práctica: "mi carrera es perito agrónomo, tema agronomía y agrícola 100\%" (10.Gu-In-M). Sin embargo, es claro que estos conocimientos, particularmente en el caso de técnicos de nivel medio, son considerados limitados: "cuando yo llegué al MAGA tenía conocimiento de perito agrónomo, cuestiones bajas, en el MAGA, gracias a Dios fui mejorando" (2.Gu-In-H). En contraste, resulta interesante mencionar que la formación recibida, si bien tenía limitaciones a nivel de conocimientos productivos, sí incluyó aspectos teóricos y experiencias prácticas vinculadas con el trabajo de ER: "había un ramo especial, de extensión rural. Nos 
preparaban en la parte teórica, después llegamos a la comunidad [...] llegamos a dar como transferencia tecnológica" (14.Ch-Gr-H).

\section{Formación de Grado Universitaria y sus Limitaciones}

Los entrevistados que poseen título de grado universitario suelen señalar que la base del conocimiento que usan para trabajar como extensionistas proviene de su formación universitaria: “[los] fundamentos básicos los trae uno de la universidad" (7.Gu-In-H), "la universidad me dio los conocimientos" (7.Ar-In-H). Sin embargo, es muy importante notar que el conocimiento que ofrecen las universidades es percibido como limitado o insuficiente para el trabajo de ER: "no es que en la universidad te enseñan todo" (5.Ec-In-M).

Pueden identificarse dos tipos claramente diferenciados de limitaciones en la formación universitaria para el trabajo de ER, uno referido a contenidos, y otro al modo de enseñanza y a los enfoques predominantes en la formación. En cuanto a las limitaciones en torno a los contenidos, algunos entrevistados señalan que la universidad no los formó en áreas técnicas específicas que luego necesitaron: "cuando yo salí de la universidad fui a trabajar en florícolas, [...] yo no sabía nada de flores” (13.Ec-Gr-M). No obstante, son muchos más los extensionistas con título en ciencias agropecuarias los que comentan que la universidad no los formó (o lo hizo de manera insuficiente) en lo que es ER, relacionamiento con los productores o temas vinculados, como género, fortalecimiento organizacional y manejo de grupos. En palabras de los propios entrevistados: "no nos dan fortalecimiento organizacional, como también un poco de pedagogía, cómo capacitar a la gente" (7.Ec-In-M), “en la universidad no te enseñan cómo hacer extensionismo, cómo llegar al agricultor" (8.Ch-In-H).

En términos porcentuales, y teniendo exclusivamente en cuenta a egresados de ciencias agropecuarias, se observan diferencias importantes entre países, ya que el problema de la falta de formación en ER y temas relacionados ha sido mencionado con particular interés en Uruguay (76\% de la muestra) y Argentina (60\%), con una presencia más baja en Guatemala (29\%), Ecuador (26\%) y Chile (20\%), sin que existan referencias en Cuba. Para interpretar estos porcentajes, téngase en cuenta que se trata de comentarios espontáneos sobre el tema, ya que el cuestionario no tuvo una pregunta específica sobre este aspecto. Por otra parte, también resulta importante evitar considerar que porcentajes más altos implican la existencia de mayores problemas a nivel formativo, en tanto la lectura de las entrevistas parece indicar mayor conciencia de la problemática, algo que puede relacionarse con otros factores como requerimientos institucionales específicos en áreas como fortalecimiento organizacional, o modelos de ER no centrados en el asesoramiento productivo.

La segunda limitación de la educación universitaria refiere al modo de enseñanza o a los enfoques predominantes en la formación. El problema más mencionado por los entrevistados que poseen título profesional es el exceso de teoría y/o la falta de práctica en la formación, sea específicamente en la materia Extensión Rural (o su equivalente) o en la carrera en general. Este problema es mencionado por el 31\% de la muestra, pero con diferencias entre países, refiriéndose con más frecuencia en Uruguay (55\%), seguido por Ecuador (33\%) y Argentina (32\%), y finalmente por Guatemala (22\%), Cuba (17\%) y Chile (10\%). Los entrevistados lo explican así: "la universidad me dio los conocimientos [...] lo que puedo reclamarle es que no tuve la suficiente práctica” (7.Ar-In-H), "cuando yo salí de la universidad, sí tenía mucho conocimiento de esto, de lo otro, pero la práctica es otra cosa" (13.Cu-Gr-H).

En paralelo, los entrevistados también plantean como segundo problema en los enfoques de la universidad la orientación hacia modelos productivos únicos, caracterizados por relaciones causales simples entre variables de nivel productivo, lo que los hace muy limitados para evaluar procesos y dinámicas complejas que incluyen factores no productivos, o para trabajar en situaciones donde la disponibilidad de recursos económicos es limitada. 
A nivel global, esta problemática fue mencionada espontáneamente por el $20 \%$ de los egresados de ciencias agropecuarias, con presencia casi exclusiva de entrevistados de Argentina (53\%), Uruguay (35\%) y Guatemala (29\%). Los extensionistas expresan este problema de diversas maneras. Por ejemplo "al inicio uno quizá lleva ya los principios básicos de la universidad, y muchas veces no... no están acorde a la realidad de las comunidades rurales" (7.Gu-In-H), "con respecto a lo técnico... [uno] se encuentra con realidades muy diversas en las cuales no tenés la posibilidad de aplicar la técnica” (5.Ar-In-H). A veces, esto se expresa como una orientación de la formación hacia grandes productores, pero este sería solo un caso particular, ya que en realidad la referencia más importante (y que se repite más frecuentemente) es el desconocimiento de la dimensión social de los procesos y de cómo debe intervenirse en situaciones con limitada disponibilidad de recursos económicos (algo frecuente en agricultura familiar o no empresarial).

\section{Formación en Extensión Rural y Prácticas en las Carreras de Ciencias Agropecuarias}

A partir de lo analizado hasta el momento, se observa que las materias de ER o sus equivalentes, así como las prácticas con productores reales, constituyen aspectos fundamentales de la formación de los extensionistas, por lo que resulta conveniente abordarlos con mayor profundidad.

Los extensionistas que más mencionan haber cursado materias en el ámbito de la ER son los argentinos y uruguayos. Uno de ellos dice "extensión era una materia, que la dabas en quinto año de la universidad" (4.Ar-In-H). Existe acuerdo con que se trata de una materia que suele ubicarse al final del plan de estudio, que es de carácter cuatrimestral, y que a veces es optativa. El caso de la Facultad de Agronomía de la Universidad de la República (Uruguay) resulta el más destacado, en tanto se hace referencia a un conjunto de asignaturas relacionadas con extensión y desarrollo rural que pueden elegirse como parte de una orientación en extensión en el último tramo de la carrera, junto a otras alternativas posibles: "el haber optado por currículum de extensión me hizo optar por hacer el curso de cooperativismo, el curso de extensión rural" (6.Ur-In-H).

Según los entrevistados, la formación específica en ER en las carreras de ciencias agrarias tiene limitaciones. En particular, algunos destacan que se trata de una formación excesivamente teórica, con espacios para práctica insuficientes o inexistentes: "es algo tan abstracto, que no lo entiendo" (15.Ur-Gr-H). A la vez, otros entrevistados señalan que, al ser una formación optativa, son pocos los que la cursan: "somos el 2 o $3 \%$ que tomamos esos cursos" (14.Ur-Gr-H). Incluso, se plantea que la duración del curso es insuficiente, no se le suele dar el valor que tiene desde la propia universidad, y al estar al final de la carrera, los estudiantes ya están pensando en recibirse en lugar de en formarse.

Los extensionistas de Ecuador y Uruguay fueron los que más mencionaron la existencia de prácticas durante la cursada de carreras en ciencias agropecuarias, los primeros en el marco de trabajos de extensión universitaria, y los segundos a partir de actividades curriculares con productores. Los entrevistados ecuatorianos plantean que la extensión universitaria puede realizarse desde el inicio de la carrera y suele incluir múltiples actividades, desde arreglo de jardines en comunidades desfavorecidas hasta capacitación de productores. En el caso uruguayo se mencionan diferentes espacios de práctica o de contacto con la realidad, que también incluyen participación en proyectos de extensión universitaria, pero como algo acotado.

A la vez, varios entrevistados destacaron visitas a campo al inicio de la carrera, para que los alumnos puedan conocer qué se hace en una finca, así como actividades prácticas hacia el final, en las cuales se debe hacer el diagnóstico de un predio: "hay talleres donde se va un día a la casa del productor, se recaba datos y después se le hace un diagnóstico y una devolución con el productor y la clase" (13.Ur-Gr-M). Finalmente, 
se destaca una práctica propia de la Facultad de Veterinaria de la Universidad de la República (Uruguay), que es la realización de actividades semanales durante el último tramo de la carrera de acompañamiento a campo a veterinarios formados, para conocer el ejercicio de la profesión: "estás ahí y un día a la semana te subes al auto con un veterinario y sales [...] a lo que sea. A tomar mate, a pesar un novillo o a ir a cobrarle a uno" (15.Ur-Gr-H).

Los entrevistados tienden a señalar que los espacios de práctica resultan insuficientes, en línea con su percepción de que la formación suele ser excesivamente teórica. En paralelo, describen múltiples aprendizajes derivados de estos espacios, como ser el conocimiento de las condiciones de vida de productores reales, cómo hacer para vincularse con las personas en el marco del trabajo técnico, y la toma de conciencia de que la realidad no permite la aplicación lineal de conocimientos aprendidos a nivel teórico, todo lo que hace posible que "al final vos tengas una idea integrada de cómo actuar frente a una realidad concreta" (13-Ur-Gr-H).

Los entrevistados también presentaron un conjunto de propuestas para que la formación universitaria contribuya de mejor manera al trabajo de ER. En general, la propuesta más frecuente es la de generar, fortalecer y aumentar los espacios de práctica, a partir de diferentes formatos, incluyendo realización de diagnósticos complejos en predios de productores y pasantías con instituciones públicas y privadas. No obstante, el formato de experiencia que se propone más frecuentemente es el acompañamiento de campo a profesionales que están llevando adelante sus tareas cotidianas, a fin de adentrarse en la práctica profesional y "que te enseñe cómo funciona la realidad" (13.Ur-Gr-H). A esta propuesta central, los entrevistados también suman otras, entre las cuales puede mencionarse el fortalecimiento de los contenidos de la formación, incluyendo herramientas para pensar la realidad de manera integrada y para el abordaje pedagógico de los productores, y el diseño de formación de posgrado acorde a la tarea del extensionista.

\section{Formación de Posgrado y sus Especificidades}

Los entrevistados también destacaron los aportes de la formación de posgrado al trabajo de ER, poniendo de relieve diferentes contrastes con la formación universitaria de grado. Como aclaración metodológica, se destaca que se optó por considerar a los diplomados como formación de posgrado cuando eran dictados por instituciones universitarias, aun cuando no fuera requisito contar con título universitario para cursarlos.

En el marco del estudio, en Argentina y Uruguay se destacó el cursado de maestrías mientras que, en Guatemala, diplomados. En el resto de los países, la referencia a posgrados fue mucho menos frecuente. Respecto de las áreas de los posgrados, se destacan Desarrollo Rural o Territorial, y Extensión Rural, aunque también se mencionaron casos como Gestión de la Producción, Gestión de Proyectos Socioproductivos, Granos, Producción Animal y Agronegocios.

Del análisis de las reflexiones de los entrevistados pudieron identificarse una serie de características específicas de la formación de posgrado que la diferencian de la formación de grado y le dan una importante potencialidad.

1. La mayor parte de las personas que cursan un posgrado en Desarrollo Rural o en Extensión Rural tienen experiencia práctica en extensión, lo que genera un marco de experiencias similares que ofrece oportunidades que no se dan a nivel de grado: "hay compañeros del territorio con que podemos hablar mano a mano, por lo menos con los que estamos en cosas similares, en gobiernos departamentales, intendencias" (1.Ur-In-H).

2. Los contenidos a abordar tienen una relación directa con la práctica cotidiana, es decir, con lo que se hace todos los días, lo que le da otra profundidad y valor a los temas que se hablan, 
haciéndolos mucho más concretos (en contraste con lo que podría vivirse como una formación excesivamente teórica).

3. Los posgrados en Desarrollo Rural y Extensión Rural tienden a tener una perspectiva holística que busca captar la complejidad y diversidad de los fenómenos que se estudian: "son distintas escalas, que toman otras dimensiones porque vos vas integrando otras cuestiones, otros factores, que regulan eso. Y esto ayuda a entender un poco la dinámica de cómo se van dando distintas situaciones y los por qué" (8.Ar-In-H).

4. En los posgrados se tiende a dar un proceso de retroalimentación creativo entre la experiencia práctica cotidiana y los contenidos propios del posgrado, lo que permite reevaluar, transformar y perfeccionar la práctica a partir de los aportes de la teoría. En este marco, los aportes teóricos funcionan como marcos integradores que permite valorar críticamente el modo de actuar cotidiano: “yo, en el momento que cursaba la maestría, me replanteaba el trabajo que estaba haciendo [...] y trataba de darle una vuelta de rosca [es decir, de mejorarlo]" (4.Ar-In-H).

En breve, se observa que la formación de posgrado tiene importantes características diferenciales, lo que le permite superar la dicotomía entre teoría y práctica, así como generar procesos de aprendizaje múltiples, que no solo permiten la incorporación de nuevos conocimientos, sino que llevan a reevaluar y trasformar las prácticas. En este proceso, los intercambios que se dan entre pares ocupan un lugar fundamental.

\section{Discusión y Conclusiones}

En este estudio se arribó a diferentes resultados. En primer lugar, debe reconocerse que los extensionistas (o futuros extensionistas) no siempre inician su formación agropecuaria o rural en la universidad, ya que muchos cuentan con una trayectoria formativa previa que debe ser tenida en cuenta, dada su contribución a la práctica profesional (BOCCHICHIO, 2013). En particular, los resultados muestran que la experiencia rural de nivel familiar puede jugar un rol importante aportando conocimientos productivos prácticos y comprensión de la idiosincrasia de las poblaciones rurales. De esta manera, debe recocerse la importancia del aprendizaje experiencial (ORD, 2012) acontecido durante la niñez y adolescencia para el trabajo posterior como extensionistas, algo generalmente no reconocido ni por la educación universitaria no por las instituciones de extensión. En paralelo, los resultados también muestran el valor de la formación agropecuaria de nivel medio y terciario, las cuales suelen ofrecer conocimientos que no siempre brinda la universidad, particularmente un saber-hacer empírico general a nivel productivo, e incluso experiencia práctica en el trabajo de apoyo a familias rurales.

Es evidente que estas fuentes de conocimiento previas no quedan anuladas o superadas por la formación universitaria, en tanto usualmente abarcan cuestiones no contempladas por aquella. Por el contrario, constituyen un aporte de base experiencial que puede ser aprovechado por la universidad (MOJARRADI; KARAMIDEHKORDI, 2016), a la vez que dan cuenta de capacidades adicionales a la formación universitaria que deberían ser valoradas por las instituciones de extensión.

Por otra parte, los entrevistados coinciden con la literatura académica en que la formación de grado universitaria constituye una base de conocimientos valiosa para el trabajo de extensión (RAGASA et al., 2016; AL-ZAHRANI et al., 2017), pero que a la vez resulta insuficiente como fuente única (SULAIMAN; DAVIS, 2012; SHUKRI AL-RIMAWI et al., 2017), por lo que debe ser complementada con estrategias formativas diversas, lo que refuerza la importancia de la formación en servicio. 
En este estudio se identificaron dos tipos de limitaciones de la educación universitaria de grado para la formación de futuros extensionistas. En cuanto a las limitaciones relativas a los contenidos, los entrevistados destacaron escasa formación recibida en el área de ER, incluyendo metodologías de extensión, y capacidad para enseñar y para relacionarse con los productores, algo también indicado en la literatura científica (GBOKU; MODISE, 2008; MOVAHEDI; NAGEL, 2012). En este punto, es interesante observar que el reclamo por la falta de temáticas específicas en el currículo formativo, en general refiere a contenidos que expresan el deseo de "saber-hacer" (cómo capacitar productores, cómo enseñar, cómo hacer extensionismo), en contraste con lo que sería el requerimiento de conocimientos teóricos o conceptuales.

En paralelo, los entrevistados también mencionaron limitaciones referidas a los enfoques o encuadres formativos, en particular el exceso de teoría y la escasa formación práctica, y la tendencia a simplificar excesivamente la complejidad de los agroecosistemas, desconociendo el rol que juegan los factores sociales. La literatura académica también ha señalado la falta de formación práctica de los egresados de las ciencias agropecuarias (GBOKU; MODISE, 2008; DA ROS, 2012; MOVAHEDI; NAGEL, 2012), enfatizando que es difícil que los académicos, valorados por la publicación de artículos y por la investigación de laboratorio, se caractericen por impulsar actividades formativas de campo o aplicadas (MOJARRADI; KARAMIDEHKORDI, 2016). En un sentido más amplio, Brown y Duguid (1991) destacan que la sociedad tiende a valorar al conocimiento abstracto por sobre el empírico, por lo que no es extraño que la formación para la práctica no sea reconocida como esencial.

En Términos de la Teoría de la Acción (ARGYRIS, 1991; TAGG, 2010), estos reclamos sugieren que la formación universitaria no permitió a los entrevistados desarrollar una teoría de la acción referida el ámbito de la ER suficientemente compleja como para abordar la práctica de manera efectiva en su diversidad y complejidad. Así, se observa que la formación universitaria en el ámbito de las ciencias agropecuarias estaría generando teorías de la acción excesivamente simplistas, lo que limitaría la posibilidad de los futuros profesionales para analizar relaciones complejas entre factores, incluyendo determinantes que exceden la dimensión estrictamente productiva. Esta reflexión resulta consistente con la literatura científica que señala la dificultad que enfrentan los estudiantes y egresados de las ciencias agropecuarias para integrar conocimientos a fin de comprender la complejidad de los agroecosistemas (SARANDÓN; FLORES, 2014) y poder actuar en contextos reales (SALOMONSSON et al., 2009), en lo que constituye una crítica de nivel epistemológico a la visión positivista del conocimiento agropecuario.

Visto lo anterior, resulta de interés reflexionar sobre la dinámica y el impacto de los espacios de práctica existentes en la formación universitaria. El ciclo del aprendizaje experiencial (ORD, 2012), evidencia que el desarrollo de capacidades para la acción requiere tomar distancia y reflexionar sobre la práctica. No obstante, esto no resulta posible cuando los espacios de práctica son inexistentes, ya que la reflexión teórica pierde el objeto sobre el que debe reflexionar. En contraste, los entrevistados sugieren que la práctica contribuye a generar una comprensión más compleja de la realidad concreta en la que se debe trabajar. En términos de la Teoría de la Acción, esto hace referencia procesos recursivos en los cuales los sujetos ajustan y complejizan las teorías que usan para ordenar sus prácticas a partir de reflexionar sobre las limitaciones de sus supuestos, conocimientos y creencias para alcanzar resultados satisfactorios en campo. De esta forma, debe reconocerse la potencialidad de los espacios de práctica en escenarios reales para integrar conocimientos y generar formas complejas de comprender la realidad, especialmente cuando se complementan con procesos reflexivos (LANDINI; BRITES, 2018).

Ahora bien, las limitaciones identificadas en la formación universitaria, al influir negativamente en el desarrollo de las capacidades de los futuros extensionistas, generan perjuicios importantes a nivel de desarrollo de los países. Para actuar sobre estos limitantes, se observa que la ampliación de contenidos constituye la alternativa más accesible, en tanto solo requiere una modificación curricular, incluyendo más conocimientos relativos al "saber-hacer". Por su parte, la incorporación de más espacios de formación 
práctica parece ser más difícil, ya que implica superar tradiciones docentes y sistemas de evaluación que priorizan el conocimiento teórico, aun cuando la existencia de iniciativas y experiencias valiosas en diferentes universidades constituye un facilitador importante. Por último, las transformaciones orientadas a desarrollar una visión sistémica compleja de los agroecosistemas parecen las más difíciles de alcanzar, dado que se requieren transformaciones profundas de nivel epistemológico. No obstante, los resultados de esta investigación sugieren la potencialidad (tanto para docentes como para alumnos) de espacios de práctica reflexivos como forma de integrar conocimientos y generar teorías de la acción más complejas para comprender los escenarios socio- productivos en lo que tienen o tendrán que desempeñarse los extensionistas.

En este punto, cabría preguntarse si los cambios propuestos son legítimos, en tanto que es discutible que las facultades de ciencias agropecuarias sean las responsables de formar extensionistas rurales. No obstante, esta discusión pierde relevancia cuando se toma conciencia que las propuestas anteriores se orientan a fortalecer las capacidades de los egresados en general, a partir de desarrollar su capacidad de acción en contextos reales de práctica.

Por otra parte, diferentes autores han señalado el valor de la formación de posgrado para el trabajo de ER (CUEVAS REYES et al., 2014; NETTLE et al., 2018). En esta investigación se comprobó la potencialidad de la formación de posgrado para superar varias de las limitaciones de la formación de grado, a partir de facilitar procesos de articulación entre teoría y práctica, y de aportar al desarrollo de abordajes holísticos y complejos, al facilitar el intercambio entre pares que comparten una misma práctica (LANDINI et al., 2017). En particular, debe reconocerse que la formación de postgrado genera una oportunidad única para tomar distancia de la práctica y reflexionar sobre ella (en línea con la propuesta del aprendizaje experiencial), con el apoyo de los contenidos teóricos abordados a nivel formativo. En este contexto, los estudiantes tienen la posibilidad de analizar críticamente sus propias teorías de la acción, integrando conocimientos y generando formas de comprensión más complejas sobre su campo de acción, aprovechando las vivencias y reflexiones de pares experimentados. Visto lo anterior, debe reconocerse el valor de los posgrados en el perfeccionamiento de los extensionistas, aunque sin olvidar que su alcance es limitado, al alcanzar solo un porcentaje pequeño de quienes hacen ER en América Latina.

En el contexto de las limitaciones de la formación universitaria como base para la práctica de la ER, diferentes autores han sugerido focalizar en la formación en servicio (SULAIMAN; DAVIS, 2012). No obstante, resulta claro que una acción integral también requiere fortalecer el sistema de educativo formal (RAGASA et al., 2016). De esta investigación derivan diferentes propuestas para fortalecer la formación que ofrecen las universidades para el trabajo de ER:

- Los resultados del estudio evidenciaron la importancia de ampliar los contenidos formativos de las carreras de ciencias agropecuarias para que incorporen conocimientos suficientes en el ámbito de la extensión, la transmisión de conocimiento y el relacionamiento con productores, lo que puede implicar la creación de nuevas asignaturas. Esto incluye revalorizar la materia Extensión Rural y sus equivalentes, asegurando que cuente con contenidos amplios y actualizados, y tenga una duración acorde con ellos. Queda por reflexionar también en qué momento de la carrera ésta resulta más apropiada, ya que ubicarla en el trayecto final parece reducir su potencialidad para ampliar la perspectiva de los estudiantes. En la misma línea, siguiendo la experiencia de la Universidad de la República (Uruguay), la implementación de un trayecto formativo final optativo en extensión y desarrollo rural (en paralelo a otros) puede constituir una alternativa de gran interés.

- La escasez de espacios de práctica en contextos reales en la formación universitaria de los extensionistas rurales también evidencia el valor de las instancias de práctica o de contacto de los estudiantes con la realidad socioproductiva, tanto para acercarse a su diversidad y complejidad, 
como para desarrollar capacidades para actuar en dichos contextos. En este sentido, el caso ecuatoriano evidencia el valor que puede tener la extensión universitaria, mientras que el caso uruguayo muestra el interés de generar procesos de diagnóstico de predios reales en diálogo con los propios productores, algo también señalado por Da Ros (2012) en Brasil. Finalmente, siguiendo propuestas de los entrevistados, el acompañamiento a profesionales que realizan sus actividades cotidianas (como se comentó en la Facultad de Veterinaria de la Universidad de la República, Uruguay), así como la realización de pasantías, se reconocen como alternativas destacadas. Cabe señalar que la realización de pasantías también ha sido mencionada en la bibliografía como una alternativa de interés para fortalecer los espacios de práctica en la formación universitaria en el ámbito agropecuario (MOVAHEDI; NAGEL, 2012; MOJARRADI; KARAMIDEHKORDI, 2016).

- Si bien se reconoce que solo un porcentaje de los extensionistas tendrá acceso a formación de posgrado, los resultados de este estudio muestran la gran potencialidad de maestrías y diplomados en extensión, desarrollo rural y temáticas similares relacionadas específicamente con la práctica de extensión. En esta línea, se destaca el valor de generar estas instancias formativas y favorecer su cursada por parte de extensionistas, incluso el diseño de diplomados que permitan la participación de extensionistas con título terciario.

- Por último, a nivel epistemológico, los resultados muestran la importancia de impulsar la discusión sobre el enfoque científico que suele sustentar la formación en ciencias agrarias, dadas sus limitaciones para pensar la complejidad de los agroecosistemas. En este sentido, se considera central que las universidades permitan reconocer a los alumnos tanto los impactos indirectos o invisibles de las tecnologías productivas utilizadas, como la incidencia del factor humano en el funcionamiento de los sistemas productivos.

A diferencia de otros trabajos que se apoyan en experiencias puntuales o que abordan el tema desde una perspectiva reflexiva, el presente estudio, apoyado en una muestra amplia de extensionistas de diferentes países de América Latina, permitió identificar y analizar varias limitaciones de la formación de grado universitaria para la tarea de ER. En este proceso, también se valorizó el rol de la formación de posgrado y se presentaron diferentes propuestas para superar estas limitaciones apoyadas en la experiencia de los propios entrevistados y en el análisis de los resultados. No obstante, es conveniente recordar que la naturaleza cualitativa del estudio no permite generalizar los resultados ni a los países en su conjunto ni a las diferentes instituciones universitarias, teniendo en cuenta que existe una gran diversidad de casos, perfiles y situaciones. En ese sentido, estos resultados deben tenerse como guías para analizar y reflexionar sobre situaciones concretas. Hacia futuro, resultará valioso complementar estos resultados con estudios de tipo cuantitativo que incluyan muestras más amplias, o con estudios cualitativos que amplíen los actores entrevistados, incluyendo a docentes de grado y posgrado.

\section{Referencias}

AL-ZAHRANI, K. et al. Assessing the competencies and training needs of agricultural extension workers in Saudi Arabia. Journal of Agricultural Science and Technology, Tehran, v. 19, n. 1, p. 33-46, 2017.

ARGYRIS, C. Teaching smart people how to learn. Harvard Business Review, Brighton, n. 69, p. 99-109, May-June 1991. 
BIANQUI et al. Reflexiones en torno a un campo posible: Psicología, extensión y desarrollo rural. In: LANDINI, F. (ed.). Hacia una psicología rural latinoamericana. Buenos Aires: CLACSO, 2015, p. 251-267.

BOCCHICCHIO, A. Perfil profesional en el área de extensión y desarrollo rural. In: FEITO, M. et al. (ed.). Contribuciones internacionales al desarrollo local y rural: Hacia una agenda de investigación. San Justo: Universidad Nacional de La Matanza, 2013, p. 184-188.

BROWN, J.; DUGUID, P. Organizational learning and communities of practice. Toward a unified view of working, learning, and innovation. Organization Science, Ithaca, NY, v. 2, n. 1, p. 40-57, 1991. https://doi. org/10.2307/2634938

CHRISTOPLOS, I.; SANDISON, P.; CHIPETA, S. Guide to evaluating rural extension. Lindau: GFRAS, 2012.

CUEVAS REYES, V. et al. Análisis del capital humano proveedor de la asistencia técnica pecuaria en Sinaloa. Región y Sociedad, Hermosillo, v. 26, n. 59, p. 151-182, 2014.

DA ROS, C. A contribuição das visitas de campo no ensino das ciências agrárias na UFRRJ. Revista Ciência em Extensão, São Paulo, v. 8, n. 1, p. 107-122, 2012.

GBOKU, M. L. S.; MODISE, O. M. Basic extension skills training (BEST): A responsive approach to integrated extension for rural development in Botswana. International Journal of Lifelong Education, London, v. 27, n. 3, p. 315-331, 2008. https://doi.org/10.1080/02601370802047817

GOMBE, S. et al. Extension worker competencies needed for effective management of self-help groups (SHG) in Gombe State. International Journal of Education and Training, Las Vegas, NV, v. 1, n. 1, p. 1-7, 2015.

KHALIL, A. H. O. et al. Human resource development competencies as predictors of agricultural extension agents' performance in Yemen. Human Resource Development International, London, v. 12, n. 4, p. 429-447, 2009.

KRAKAUER, P.; DOS SANTOS, S.; ALMEIDA, M. Teoria da aprendizagem experiencial no ensino de empreendedorismo: Um estudo exploratório. REGEPE - Revista de Empreendedorismo e Gestão de Pequenas Empresas, São Paulo, v. 6, n. 1, p. 101-127, 2017. https://doi.org/10.14211/regepe.v6i1.353

LANDINI, F. Necesidades formativas de los extensionistas rurales paraguayos desde la perspectiva de su función, sus problemas y sus intereses. Trabajo y Sociedad, Santiago del Estero, n. 20, p. 149-160, 2013.

LANDINI, F. Concepción de "extensión rural" de los extensionistas rurales argentinos que trabajan en el sistema público nacional con pequeños productores. Cuadernos de Desarrollo Rural, Bogotá, v. 12, n. 75, p. 33-53, 2015.

LANDINI, F.; BRITES, W. Evaluation and impact of a reflective training process for rural extension agents. The Journal of Agricultural Education and Extension Wageningen, v. 24, n. 5, p. 457-472, 2018. https:// doi.org/10.1080/1389224X.2018.1500922

LANDINI, F.; BRITES, W.; MATHOT, M. Towards a new paradigm for rural extensionists' in-service training. Journal of Rural Studies, Oxford, v. 51, p. 158-167, 2017. https://doi.org/10.1016/j.jrurstud.2017.02.010

LEFORE, N. Strengthening facilitation competencies in development: Processes, challenges and lessons of a learning alliance to develop facilitators for local community engagement. Knowledge Management for Development Journal, London, v. 11, n. 1, p. 118-135, 2015. 
MOJARRADI, G.; KARAMIDEHKORDI, E. Factors influencing practical training quality in Iranian agricultural higher education. Journal of Higher Education Policy and Management, Melbourne, v. 38, n. 2, p. 183-195, 2016. https://doi.org/10.1080/1360080X.2016.1150549

MOSCHITZ, H. et al. Learning and innovation networks for sustainable agriculture: Processes of co-evolution, joint reflection and facilitation. The Journal of Agricultural Education and Extension, Wageningen, v. 21, n. 1, p. 1-11, 2015. http://doi.org/10.1080/1389224X.2014.991111

MOVAHEDI, R.; NAGEL, U. Identifying required competencies for the agricultural extension and education undergraduates. Journal of Agricultural Science and Technology, Tehran, v. 14, n. 4, p. 727-742, 2012.

MULDER, M. Interdisciplinarity and education: Towards principles of pedagogical practice. The Journal of Agricultural Education and Extension, Wageningen, v. 18, v. 5, p. 437-442, 2012. https://doi. org/10.1080/1389224X.2012.71046

NETTLE, R.; CRAWFORD, A.; BRIGHTLING, P. How private-sector farm advisors change their practices: An Australian case study. Journal of Rural Studies, Oxford, v. 58, p. 20-27, 2018. https://doi.org/10.1016/j. jrurstud.2017.12.027

ORD, J. John Dewey and experiential learning: Developing the theory of youth work. Youth \& Policy, Berlin, n. 108 , p. 55-72, 2012.

PEIXOTO, A.; PEREIRA, R. Discurso versus ação no comportamento ambientalmente responsável. Revista de Gestão Ambiental e Sustentabilidade, São Paulo, v. 2, n. 2, p. 71-113, 2013.

RAGASA, C. et al. Factors affecting performance of agricultural extension: Evidence from Democratic Republic of Congo. The Journal of Agricultural Education and Extension, Wageningen, v. 22, n. 2, 113143, 2016. https://doi.org/ 10.1080/1389224X.2015.1026363

SALOMONSSON, L. etal. Farming systems education: Case study of Swedish test pilots. Renewable Agriculture and Food Systems, New York, v. 24, n. 1, p. 48-59, 2009. https://doi.org/ 10.1017/S1742170508002408

SARANDÓN, S.; FLORES, C. Agroecología: bases teóricas para el diseño y manejo de agroecosistemas sustentables. La Plata: Universidad Nacional de La Plata, 2014.

SHARMA, A.; EZUNG, P.; SHARMA, R. Impact of pineapple cultivation training on village extension officers. International Journal of Multidisciplinary Approach \& Studies, Bhopal, v. 3, n. 1, p. 6-12, 2016.

SHUKRI AL-RIMAWI, A.; ALLAHYARI, M.; AL-RUSHEIDAT, J. Assessing extension agent training needs, barriers and training methods in Jordan. Journal of Agricultural Science and Technology, Tehran, v. 19, n. 5, p. 1019-1029, 2017.

SINGH, N. et al. Impact of training programs of Extension Education Institute Nilokheri on master trainers of State Agricultural University. Agricultural Science Digest, Karnal, v. 30, n. 4, p. 254-257, 2010.

SULAIMAN, R.; DAVIS, K. The "new extensionist”: Roles, strategies, and capacities to strengthen extension and advisory services. Lindau: GFRAS, 2012.

TAGG, J. The learning-paradigm campus: From single- to double-loop learning. New Directions for Teaching and Learning, San Francisco, n. 123, 51-61, 2010. https://doi.org/10.1002/tl.409 
TAYLOR, S.; BOGDAN, R. Introducción a los métodos cualitativos de investigación. Buenos Aires: Paidós, 1990.

TORRADO PORTO, R.; CATULLO, J. Extensión rural y enfoque territorial: Aprendiendo en la acción con otros. Revista de la Facultad de Agronomía, Buenos Aires, v. 116, p. 19-27, 2017.

YARDLEY, S.; TEUNISSEN, P.; DORNAN, T. Experiential learning: Transforming theory into practice. Medical Teacher, Abingdon, v. 34, n. 2, p. 161-164, 2012. https://doi.org/ 10.3109/0142159X.2012.643264

\section{Sobre el Autor}

Fernando Landini es licenciado y doctor en Psicología por la Universidad de Buenos Aires. Investigador Independiente del Consejo Nacional de Investigaciones Científicas y Técnicas (CONICET). Estudia procesos de extensión rural e innovación agropecuaria desde una perspectiva psicosocial.

Recibido: 14 ene. 2019

Aceptado: 27 ago. 2020 